\title{
Stimulus-naming effects at different stages of motor paired-associate learning*
}

\author{
JOAN H. CANTOR \\ Institute of Child Behavior and Development, University of Iowa, Iowa City, Iowa 52240
}

\begin{abstract}
In a combined verbal and motor paired-associate task, nonsense names and button-pushing responses were learned for three random shapes, with verbal training beginning after 3,12 , or 24 motor learning trials. Earlier studies indicated that concurrent verbal and motor learning produce a strong tendency for the naming and button-pushing responses to be learned in "consistent" pairs. According to the theoretical analysis presented, the verbal-motor consistency is explained by the development of name-button associations. It was predicted that these associations affect both motor errors and correct responses, that their effects are greater for correct responses, and that attenuation of the effects due to delay in verbal training is greater for motor errors. The results are in good agreement with the predictions, and they suggest that name-button associations develop rapidly even under delayed verbal training.
\end{abstract}

Stimulus naming has been shown to affect discrimination learning in a wide variety of experiments, including studies of both acquired distinctiveness and acquired equivalence of cues (see Arnoult, 1957; Cantor, 1965; Spiker, 1956a, 1963; for literature reviews). More recently, interest has centered on the effects of dimensional naming responses in discrimination tasks such as simultaneous discrimination learning (Spiker, 1971), reversal and nonreversal shift learning (Kendler \& Kendler, 1961; Silverman, 1966; Guldmann, 1972), and optional shift learning (Kendler, 1964; Lundback, 1971). S-R explanations of the facilitative .and interfering effects of stimulus naming in these situations have frequently assumed that performance in the discrimination task is a function, at least in part, of associations that are learned between the names and the criterion responses. Since these hypothesized associations play such a significant explanatory role, it is important to be able to specify the variables that affect their development. The author has reported a method for determining the effects of experimental manipulations on the strength of these associations, through the use of overt naming responses in a motor paired-associate task (Cantor, 1970). In the experimental task, names and button-pushing responses are learned concurrently for a set of three similar random shapes, and the effects of name learning on motor performance are assessed through the use of conditional probabilities.

In the earlier experiments (Cantor, 1970), it was demonstrated that the verbal and motor responses tend to be learned in pairs, with the result that the naming

*The research reported here was supported in part by U.S. Public Health Service Grant HD-01376, National Institute of Child Health and Human Development. The author is indebted to Charles C. Spiker for many valuable suggestions in the preparation of the manuscript. Thanks are also due to Ann Bigelow, Thomas Seif. and Janet McKee for collecting the data and to Harriette Guldmann for assistance in analyzing the data. responses both facilitate and interfere with motor performance in the same task. More specifically, the Ss show a strong tendency to make motor responses that are consistent with the stimulus names they use. Thus, if the naming response is correct, the probability of a correct motor response is very high. Furthermore, if the stimulus is named incorrectly, the Ss tend to make a motor error consistent with the name given. For example, on a trial when Stimulus 1 is presented and $S$ gives the name appropriate for Stimulus 2 , there is a strong probability that he will also push the button appropriate for Stimulus 2 and thus make a "consistent" error. The earlier studies also indicated that the tendency to respond with consistent pairs of verbal and motor responses develops very rapidly under conditions of concurrent verbal and motor learning, with a faster rate of development for names that are both distinctive and familiar to the $\mathrm{S}$. The development of strong name-button response pairs is consistent with the hypothesis that verbal-motor associations are an important factor in the discrimination performance. Furthermore, the rapid rate of development of the pairings is consistent with related findings that discrimination learning occurs rapidly under certain naming conditions. For example, new learning in reversal and nonreversal shift tasks is very rapid under conditions where Ss are naming values on the relevant dimension (Kendler \& Kendler, 1961: Silverman, 1966; Guldmann, 1972).

The purpose of the present experiment was to determine whether the rate of development of the verbal-motor response pairs is a function of the stage of motor learning in which the verbal training begins. In many discrimination tasks, it is hypothesized that Ss change naming responses either in later stages of acquisition or at the beginning of a transfer task. It is therefore important to know whether consistent name-button response pairs develop under conditions of 


\section{CORRECT ASSOCLATIONS}

W VERBAL-MOTOR TASK
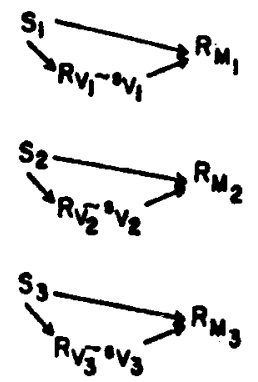

ANALYSIS OF CONSISTENT MOTOR RESPONSES
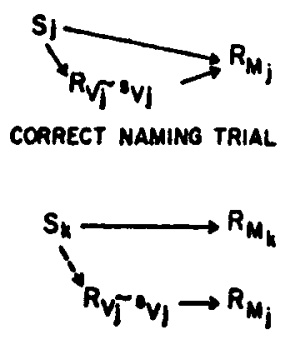

INCORRECT NAMING TRIAL

Fig. 1. An S-R analysis of learning in the verbal-motor task.

delayed verbal training and, if so, whether their rate of development depends on the amount of delay. In the present experiment, similar names (GEK, KEZ, ZEG) and button-pushing responses were learned for a set of three similar random shapes. Verbal training began after 3 prior motor trials (stimulus presentations) for one-third of the Ss (Group V3), after 12 motor trials for another third (Group V12), and after 24 motor trials for the remaining third (Group V24). Each $S$ also received motor training alone with a second set of three random shapes so that the general effects of naming on motor performance could be assessed on a within- $S$ basis. The specific theoretical questions of interest will be discussed following presentation of an S-R analysis of learning in the verbal-motor task.

The analysis of concurrent learning of verbal and motor responses to the three random shapes is presented on the left side of Fig. 1. Three types of correct S-R associations that are hypothesized to develop (figure-button, figure-name, and name-button) are diagrammed for each stimulus. Verbal performance on each trial is assumed to depend on the strength of the correct figure-name association, e.g., $S_{1} \rightarrow R_{V_{1}}$, relative to that of competing incorrect associations, such as $\mathrm{S}_{1} \rightarrow \mathrm{R}_{\mathrm{V}_{2}}$. (Dotted arrows represent incorrect associations; for clarity, only correct associations are included in this part of the diagram.) Each verbal response is assumed to produce distinctive stimulus feedback to the $S$, thereby adding a verbal response-produced cue to the stimulus complex. As a result, two correct motor response tendencies presumably develop: a figure-button association (e.g., $S_{1} \rightarrow R_{M_{1}}$ ), and a name-button association (e.g., $\mathrm{sV}_{1} \rightarrow \mathrm{R}_{\mathrm{M}_{1}}$ ). The tendency to make a correct motor response on a particular trial is a function of the joint strength of these two associations. Furthermore, incorrect associations of either type (figure-button or name-button) may compete with the correct response tendencies on a particular trial.

This theoretical analysis is used to explain the observed consistency in verbal-motor response pairs in

the following way. If we let $R_{V_{j}}$ and $R_{M_{j}}$ represent the correct verbal and motor responses to a particular stimulus $S_{j}$, then $R_{M_{j}}$ is designated as a consistent motor response for any trial in which it follows the occurrence of $R_{V_{j}}$. Thus, the consistent motor response $R_{M_{j}}$ may be either correct or incorrect for the stimulus presented. A consistent correct response occurs any time S's verbal and motor responses are both correct, e.g., $S_{1}$ is presented and $R_{V_{1}}$ occurs followed by the consistent response $\mathbf{R}_{\mathbf{M}_{1}}$. A consistent error in the same example would be $R_{V_{2}}$ followed by $R_{M_{2}}$. (A nonconsistent correct response would be $R_{V_{2}}$ followed by $R_{M_{1}}$, and a nonconsistent error would be $R_{V_{2}}$ followed by $R_{M_{3}}$.) The associations assumed to produce both types of consistent responses are diagrammed on the right side of Fig. 1. It should be noted that on correct naming trials, the two associations $S_{j} \rightarrow R_{M_{j}}$ and $s_{V_{j}} \rightarrow R_{M_{j}}$ are not competing tendencies, but rather they combine to produce a stronger tendency for the correct motor response to occur. On incorrect naming trials, $S_{k} \rightarrow R_{M_{k}}$ and $s_{j} \rightarrow R_{M_{j}}$ are competing response tendencies, and if the latter association is sufficiently strong, a consistent motor error occurs.

\section{THEORETICAL PREDICTIONS}

If this theoretical analysis is correct, the development of name-button associations should produce the following effects on motor performance in the present experiment.

(1) The observed proportion of total consistent motor responses (consistent correct responses and errors) should be greater than a probability computed under an assumption of verbal-motor independence, i.e., $\mathrm{P}\left(\mathrm{M}_{\mathrm{tcs}}\right)$ $>\mathrm{P}\left(\mathrm{M}_{\mathrm{tcs}}\right)_{\text {ind }}$. The latter probability assumes no development of $s_{V} \rightarrow R_{M}$ associations, but rather attributes any increase in consistent motor responses over trials to the concurrent but independent strengthening of correct $S \rightarrow R_{V}$ and $S \rightarrow R_{M}$ associations. (Methods of computing the various response measures will be described in a later section.)

(2) The probability of a consistent motor response should be greater on correct naming trials than on incorrect naming trials, i.e., $P(M \mid V)>P\left(\bar{M}_{c s} \mid \bar{V}\right)$. This difference is predicted because, on correct naming trials, the name-button association $s_{V_{j}} \rightarrow R_{M_{j}}$ combines with $S_{j} \rightarrow R_{M_{j}}$ to produce the consistent correct motor response. On incorrect naming trials, ${ }^{s} V_{j} \rightarrow R_{M_{j}}$ must be stronger than $S_{k} \rightarrow R_{M_{k}}$ to produce a consistent motor error (see the right side of Fig. 1).

(3) On naming-error trials, the conditional probability of a consistent motor error should be greater than the comparable probability of a nonconsistent error, i.e., $P\left(\bar{M}_{c s} \mid \bar{V}\right)>P\left(\bar{M}_{n c s} \mid \bar{V}\right)$. If name-button associations are not learned, these two probabilities should be equal.

(4) Delay in verbal training should have relatively little effect on consistent correct responses, $P(M \mid V)$, since the newly developing name-button associations can 
increase this probability even when the figure-button associations are already relatively strong. On the other hand, delay in verbal training should result in a lower probability of consistent errors, $\mathrm{P}\left(\overline{\mathrm{M}}_{\mathrm{cs}} \mid \overline{\mathrm{V}}\right)$, since the name-button associations must gain considerably in strength before they can compete successfully with the previously strengthened figure-button associations. If this reasoning is correct, delay in verbal training should increase the difference predicted in (2) above and should decrease the difference predicted in (3). Furthermore, the highly similar names used in the present study should maximize the predicted effects of delay, since the name-button associations appear to develop more slowly with similar than with distinctive names (Cantor, 1970).

\section{METHOD}

\section{Design and Subjects}

Initially, motor training alone was given to each $S$ for a set of three white random shapes and, on alternate trials, for a set of three black shapes. The combined verbal-motor (experimental) task began at the point in motor training appropriate for the particular verbal group. Following a brief instructional interval, $S$ began to learn names (GEK, KEZ, ZEG) in addition to the button-pushing responses for one of the stimulus sets. Motor training alone continued with the control set of stimuli.

The use of three verbal conditions and the counterbalancing of stimulus sets with respect to the experimental and control tasks resulted in a 3 by 2 factorial design. The Ss were 120 boys and girls, ages 13.16 , who were each paid $\$ 1.50$ for volunteering to serve in the experiment. Twenty Ss were assigned randomly to each of the six groups.

\section{Stimuli and Apparatus}

The stimuli and apparatus were similar to those used in the earlier studies and are described here in somewhat less detail. The stimuli were the same two sets of 24-point random shapes reproduced in the earlier report (Cantor, 1970, Experiment 2). These shapes were selected from two sets that Somnapan (1968) developed. using a method of systematic variation of a prototype figure. The black figures constituted the experimental set and the white figures the control set for half the $\mathrm{Ss}$, the reverse being true for the remaining Ss. Each name was arbitrarily assigned to a specific stimulus in each set.

A Kodak Carousel random-access projector was used to present the stimuli. The image was projected on the rear surface of a thin translucent viewing screen. The $S$ sat in front of the screen and viewed the stimuli at approximately eye level. Each figure appeared centered in a $5-\mathrm{cm}$ square of contrasting brightness. The stimulus alone appeared during the anticipation interval, and a chime sounded if $S$ pushed the correct button. During the joint-presentation interval, the stimulus was repeated either alone (control stimulus) or with its associated name printed in block letters underneath it (experimental stimulus).

The six response buttons were arranged in a semicircle (radius of $9.5 \mathrm{~cm}$ ) below the screen on a panel that slanted toward the $S$. Three black buttons were located on the left side, and each was assigned to a particular black figure. Similarly, three white buttons on the right side were assigned to the white figures. Each button had a small white light located above it that was used to identify the correct button during joint-presentation intervals. The selection of stimuli, stimulus duration, and feedback for correct responses were automatically controlled by punched-tape programming equipment and interval timers.

\section{Procedure}

The $S$ was seatad and given instructions for the motor task. The essential statements in the instructions were the following: "... A number of odd-shaped figures will be shown on the screen, one at a time. There will be three different white figures and three different black figures. Each one of these figures goes with one of the buttons on the panel.... If you push the right button, you will hear a chime. A short time after you push the button, there will be an information period. During this time, the same figure will be repeated and the light next to the correct button will come on...."

Motor training began immediately following the instructions and consisted of 96 trials (stimulus presentations) for all Ss. Control and experimental stimuli were presented on alternate trials throughout training. All Ss received the same sequence of stimuli, in which each successive block of 12 stimuli consisted of two presentations of each of the six figures. The durations of the anticipation and joint-presentation intervals were 6 and $4 \mathrm{sec}$, respectively. A $30-\mathrm{sec}$ rest period was given at the end of each block of 24 trials. Instructions for name learning required $60 \mathrm{sec}$.

Verbal training began after 3 trials with the experimental stimuli for Group V3 (i.e., following 6 trials consisting of alternating experimental and control stimulus presentations), 12 trials for Group V12, and 24 trials for Group V24. The following instructions were given: "From now on, the black (or white) figures will also have names for you to learn. When a black figure comes on, the first thing you do is guess its correct name. Then push the button that you think is correct. During the information period, the figure and its correct name will come on the screen in addition to the information light. Watch to see if you said the correct name each time..." The three names (printed on $3 \times 5$ in. cards) were shown to $S$, one at a time. $E$ first pronounced each name and then asked $S$ to repeat it out loud. The verbal-motor training began immediately following these instructions.

\section{Verbal-Motor Response Measures}

The criterion data were derived only from verbal-motor trials in which $S$ had responded with one of the three designated names and had also pushed a button within the anticipation interval. Since the names were highly similar, some extralist verbal errors occurred in the early trials; the data from these trials were not used, since they provide no information regarding the consistency of the motor responses.

The data were tabulated on an individual $S$ basis for each block of six trials, with the exception of the first trial block for Group V3. Since, for this group, verbal training began in the middle of the first six-trial block of the experimental task, the first block is based on three rather than six trials and is designated Trial Block 1B.

The response measures include frequencies of correct verbal responses (V), correct motor responses (M), trials with verbal and motor responses both correct (VM), and trials with verbal and motor responses both incorrect $(\overline{\mathrm{VM}})$. All motor responses in VM trials are consistent correct responses, i.e., $\mathrm{VM}=\mathrm{M}_{\mathrm{cs}}$. The motor responses in $\overline{\mathrm{VM}}$ trials are further categorized as either consistent errors $\left(\bar{M}_{c s}\right)$ or as nonconsistent errors $\left(\overline{\mathrm{M}}_{\text {ncs }}\right)$. These frequency measures are converted to proportions by dividing by $\mathrm{N}$, the number of trials in the block that can be scored for motor consistency, e.g., $P(V)=V / N$. The proportion of total consistent motor responses includes both correct responses and errors. i.e.. $\mathrm{P}\left(\mathrm{M}_{\mathrm{tcs}}\right)=\left(\mathrm{M}_{\mathrm{cs}}+\overline{\mathrm{M}}_{\mathrm{cs}}\right) / \mathrm{N}$. The conditional probabilities are all computed in the conventional manner, e.g. $\mathrm{P}(\mathrm{M} / \mathrm{V})=\mathrm{VM} / \mathrm{V}=$ $\mathrm{M}_{\mathrm{cs}} / \mathrm{V}$.

The computation of the probability of a consistent response. under the assumption of verbal-motor independence. involves application of the general principle that the joint probability of occurrence of independent events is the product of their separate probabilities. Thus. 

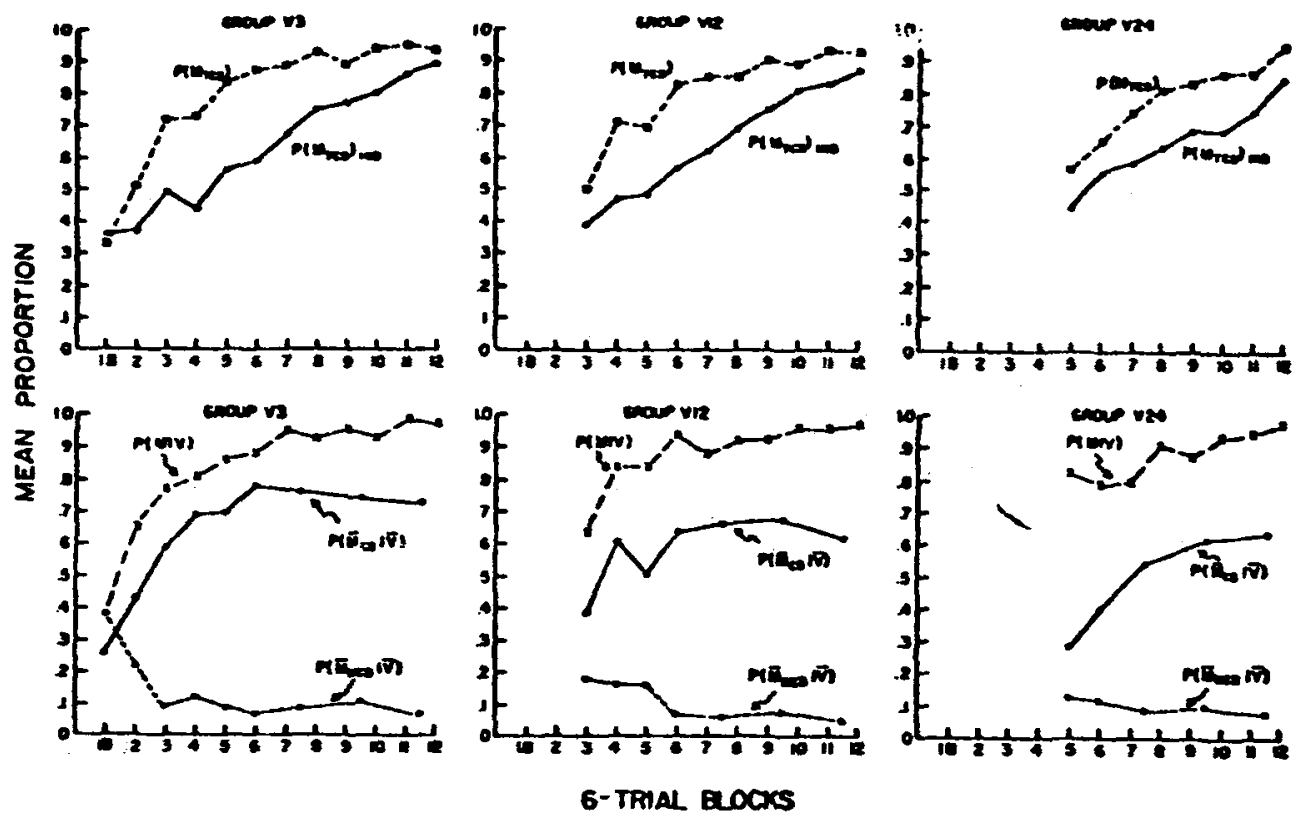

Fig. 2. Delay-group means for the various verbal-motor response measures, plotted over trial blocks.

$$
P\left(M_{t c s}\right)_{\text {ind }}=\{P(V) \times P(M)]+\frac{[P(\bar{V}) \times P(\bar{M})]}{2} .
$$

The first bracketed expression gives the probability of consistent correct responses, and the remaining expression gives the probability of consistent errors. The error portion is divided by two. since the motor errors in $\overline{\mathrm{VM}}$ trials may be either consistent or nonconsistent.

\section{RESULTS}

In all analyses of variance to be reported, stimulus set and trial blocks are included as factors in addition to the variables specifically mentioned. For clarity, only those effects relevant to the specific prediction are discussed. The probabilities associated with all $F$ ratios reported as significant are $<.005$ unless otherwise stated.

\section{Experimental vs Control Performance}

The overall effects of naming on motor performance are assessed by comparing the mean proportions of correct responses to the experimental (named) vs the control stimuli. Consistent with earlier findings, control performance is slightly better than experimental performance. The mean proportion of correct responses over all trial blocks is .68 for the control stimuli and .66 for the experimental stimuli. Analysis of variance indicates that the experimental-control difference is significant $[F(1,114)=7.35, p=.008]$ and that there is no significant interaction with amount of delay in verbal training.

\section{Verbal-Motor Performance}

The data and statistical findings relevant to each of the four predictions will be presented in turn. Although each response measure is computed for every $S$, within-S comparisons among response measures cannot be made because the measures are not independent. For this reason, in all analyses in which two response measures are compared, the data for half the $S s(N=10)$ in each group are used to compute one measure, and the data for the remaining half are used to compute the other measure. In analyses involving delay-group comparisons (V3 vs V12 and V12 vs V24), only those trial blocks are included for which both groups had verbal training.

(1) The observed probability of total consistent motor responses is expected to be greater than the probability that is based on an assumption of verbal-motor independence, i.e., $\mathrm{P}\left(\mathrm{M}_{\mathrm{tcs}}\right)>\mathrm{P}\left(\mathrm{M}_{\mathrm{tcs}}\right)_{\text {ind }}$. The group means for these measures are plotted over trial blocks in the top three graphs of Fig. 2. It can be seen that $P\left(M_{t c s}\right)$ is markedly higher than $P\left(M_{t c s}\right)_{\text {ind }}$ in all three groups. The difference is significant in each group $[F(1,36)=38.99$ for $V 3,30.21$ for $V 12$, and 12.12 for V24]. There are no significant group differences in either of the response measures, nor are the Response Measure by Groups interactions significant.

(2) The probability of a consistent motor response is expected to be greater on correct naming trials than on incorrect naming trials, i.e., $P(M \mid V)>P\left(\bar{M}_{c s} \mid \bar{V}\right)$. The data are clearly in accord with the prediction, as seen in the lower graphs in Fig. 2. The difference is significant in each group $[F(1,36)=11.90$ for V3, 17.61 for V12, and 57.33 for V24]. These analyses of variance include Trial Blocks 1B-6 for V3, Trial Blocks 3.6 for V12, and Trial Blocks 5.6 for V24. Data beyond Trial Block 6 are excluded from all analyses involving error measures because of a marked reduction in verbal errors late in training. For the same reason, the error measures are 
plotted in 12-trial blocks rather than 6-trial blocks in the last half of training.

(3) On naming-error trials, the probability of a consistent error is expected to be greater than the probability of a nonconsistent error, i.e., $P\left(\bar{M}_{c s} \mid \bar{V}\right)>$ $\mathrm{P}\left(\overline{\mathrm{M}}_{\mathrm{ncs}} \mid \overline{\mathrm{V}}\right)$. It can be seen in the lower graphs in Fig. 2 that the consistent errors increase and the nonconsistent errors decrease, as predicted. The difference is significant in each group $[F(1,36)=59.69$ for V3, 43.46 for V12, and $5.65(\mathrm{p}=.023)$ for V24]

(4) Delay in verbal training is expected to have relatively little effect on consistent correct responses, $\mathrm{P}(\mathrm{M} \mid \mathrm{V})$. There are no significant differences among the groups in this measure $[F(2,114)=1.07]$. On the other hand, delay is expected to decrease consistent errors, $\mathrm{P}\left(\overline{\mathrm{M}}_{\mathrm{cs}} \mid \overline{\mathrm{V}}\right)$. The group differences in consistent errors seen in Fig. 2 are significant $[F(2,114)=18.99]$, and follow-up tests indicate that all pairs of groups differ significantly. These predictions for the delay variable should also be reflected in interactions with groups in the analyses reported in (2) and (3) above. The difference $P(M \mid V)>P\left(\vec{M}_{c s} \mid \vec{V}\right)$ reported in (2) increases with delay, as predicted. The interaction is not significant for V3 vs V12, but is significant for V12 vs $\mathrm{V} 24[\mathrm{~F}(1,72)=4.93(\mathrm{p}=.030)]$. The difference $\mathrm{P}\left(\overline{\mathrm{M}}_{\mathrm{cs}} \mid \overline{\mathrm{V}}\right)>\mathrm{P}\left(\overline{\mathrm{M}}_{\mathrm{ncs}} \mid \overline{\mathrm{V}}\right)$ reported in (3) decreases with delay, as predicted. The interaction is marginally significant for V3 vs V12 $[F(1,72)=3.86(p=.053)]$ and clearly significant for $\mathrm{V} 12$ vs $\mathrm{V} 24[\mathrm{~F}(1,72)=6.55$ $(\mathrm{p}=.013)]$.

\section{DISCUSSION}

The present results are in close agreement with all of the theoretical predictions, and they extend the findings of the earlier studies (Cantor, 1970). The refined response measures reported here appear to be more sensitive to the effects of the experimental manipulations than were those previously employed. The results provide considerable support for concluding that (1) name-button associations contribute to motor performance, producing both facilitation and interference in the same task, and (2) these associations develop rapidly even under conditions of delayed verbal training. It should be noted that the terms facilitation and interference in this context have specific reference to the conditional probabilities of consistent correct responses and errors, respectively, rather than to overall effects of naming on motor performance. It will be recalled that overall motor performance is slightly better for the control stimuli than for the experimental stimuli, probably because of the greater complexity of the combined verbal-motor task.

The effects of delay in verbal training are of particular interest. Facilitation is very high in all delay groups. whereas interference is a decreasing function of delay. Thus, similar naming responses introduced in the early stages of the task have both strong facilitation and interference effects, whereas those introduced in the later stages produce far more facilitation than interference. These findings are consistent with the $S \cdot R$ analysis presented earlier.

Consideration of the results of all three experiments indicates that delay in verbal training and similarity of the names are both relevant variables, and that these variables appear to interact with each other. First let us consider the effects of similarity of names when there is no delay in verbal training. The names used in the present study are the same ones used in the groups given similar-name training in the earlier studies. In all cases, the similar names produce high probabilities of consistent correct responses and moderately high probabilities of consistent errors. When distinctive names are used, the probability of consistent errors is greatly increased (Cantor, 1970, Experiment 2). Thus, although the distinctive names are easier to learn, the naming errors that do occur have a much higher probability of producing motor errors. Furthermore, there is a higher probability of consistent correct responses in the early stages of learning.

Two explanations of these findings will be discussed within the present S-R framework, one in terms of stimulus generalization and the other in terms of rehearsal. Consider first the role of stimulus generalization. In the $S-R$ analysis in Fig. 1 , let us assume that similar names produce similar verbal cues and that distinctive names produce distinctive cues. When the names are similar, incorrect name-button associations (e.g., $s_{V_{1}} \rightarrow R_{M_{2}}$ ) should be relatively strong due to generalization among the similar cues. When the names are distinctive, incorrect name-button associations should remain relatively weak. As a result, consistent errors should be more frequent with distinctive names, and the earlier data clearly support this prediction. The probability of consistent correct responses should also be higher with distinctive names, although it will be recalled that the strength of the name-button associations is expected to have less effect on correct responses than on errors. This prediction is also supported early in training, before the probabilities for both groups approach unity.

The second explanation is based on the assumption that Ss tend to use the names to rehearse between trials (Spiker, 1956b). Rehearsal in the present task might take the form, "GEK goes with Button 1." Theoretically, the effect of this rehearsal is additional strengthening of the name-button associations. It seems quite plausible that correct rehearsal is more difficult with highly similar names, resulting in fewer rehearsal trials for the correct associations. The predicted effects of name similarity are therefore the same for the rehearsal and generalization hypotheses.

Next consider the effects of delay in verbal training. The only available data are those from the present study in which the names were highly similar. As noted earlier. the name-button associations appear to develop quite 
rapidly even under delayed verbal training. Recall that facilitation is virtually unaffected by the delay: that is. the delay groups do not differ in $P(M \mid V)$. Even on the first verbal-motor trial block for Group V24, P(M | V) is .83. Thus, although the probability of a naming error is still high (.64), the probability of a correct motor response in this trial block is .83 on a correct naming trial and only .52 on an incorrect naming trial. This finding strongly suggests that rehearsal is occurring, particularly in the verbal delay groups.

If these conclusions regarding rehearsal are correct, then verbal delay and name similarity would be expected to interact. If verbal training were given with distinctive rather than similar names, the more effective rehearsal should produce an extremely rapid development of both consistent errors and correct responses, thereby minimizing the effects of any delay in verbal training.

Finally, in a somewhat broader context, the results and explanations offered here are consistent with other findings in discrimination learning referred to in the introduction. In particular, the rehearsal hypothesis appears to have considerable merit in explaining the potent effects of naming responses in many types of discrimination learning and the rapidity with which certain discrimination shifts are made by older children and adults.

\section{REFERENCES}

Arnoult, M. D. Stimulus predifferentiation: Some generalizations and hypotheses. Psychological Bulletin, 1957, 54, 339-350.

Cantor, J. H. Transfer of stimulus pretraining in motor paired-associate and discrimination learning tasks. In L. P. Lipsitt and C. C. Spiker (Eds.), Advances in child development and behavior. Vol. 2. New York: Academic Press, 1965. Pp. 19-58.

Cantor. J. H. Facilitating and interfering effects of stimulus naming on children's motor paired-associate learning. Journal of Experimental Child Psychology, 1970. 10. 374-389.

Guldmann, H. The effects of dimensional verbalization upon children's performance on reversal and extradimensional shift discrimination problems. Unpublished doctoral dissertation, University of Iowa, 1972.

Kendler, H. H.. \& Kendler, T. S. Effects of verbalization on reversal shifts in children. Science, 1961, 134, 1619-1620.

Kendler, T. S. Verbalization and optional reversal shifts among kindergarten children. Journal of Verbal Learning \& Verbal Behavior, 1964, 3, 428-436.

Lundback, E. The effects of dimensional naming upon children's performance in a modified optional shift problem. Unpublished master's thesis, University of Iowa, 1971.

Silverman, I. W. Effect of verbalization on reversal shifts in children: Additional data. Journal of Experimental Child Psychology, 1966, 4, 1-8.

Somnapan, R. Development of sets of mutually equally discriminable random shapes. Journal of Experimental Psychology, 1968, 76, 297-302.

Spiker, C. C. Experiments with children on the hypothesis of acquired distinctiveness and equivalence of cues. Child Development, 1956a, 27, 253-263.

Spiker, C. C. Stimulus pretraining and subsequent performance in the delayed reaction experiment. Journal of Experimental Psychology, 1956b, 52, 107-111.

Spiker, C. C. Verbal factors in the discrimination learning of children. In J. C. Wright and J. Kagan (Eds.), Basic cognitive processes in children. Monographs of the Society for Research in Child Development, 1963, 28, No. 2, 53-69.

Spiker, C. C. Application of Hull-Spence theory to the discrimination learning of children. In H. W. Reese (Ed.), Advarices in child develupment and behavior. Vol. 6. New York: Academic Press, 1971. Pp. 99-152.

(Received for publication September 1, 1972; revision received October 18,1972 .) 